\title{
Quattro nuove sezioni AIRP e la nascita di PKD International
}

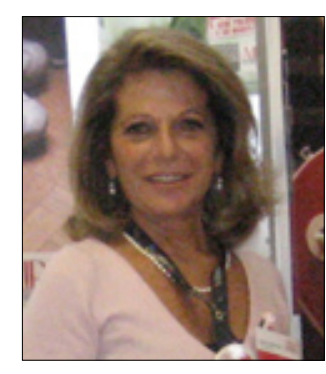

Luisa Sternfeld Pavia

Vice Presidente

Associazione Italiana Rene

Policistico AIRP onlus

Luisa.sternfeld.airp@renepolicistico.it
Cari lettori e cari amici,

è con grande piacere che vi ritrovo sulla nostra Newsletter, per tracciare insieme a voi un riepilogo delle molteplici attività di AIRP nel secondo trimestre 2011.

Il mese di marzo, come ricorderete, si era chiuso con la nostra trionfale partecipazione alla Maratona di Roma. Nemmeno il tempo di tirare il fiato e ai primi di aprile ecco l'inaugurazione di quattro Sezioni Periferiche della nostra Associazione, con i relativi responsabili, che desidero citare: per la Sezione di Montichiari, Grazia Sandrini; per la Sezione di Roma, Fabio Dal Canto; per la Sezione di Rimini, Lidia Tonini e per la Sezione di Novara Luigi Gronchi. A tutti loro va il nostro saluto e il nostro ringraziamento per l'impegno che porteranno alla causa dei pazienti.

Sempre nel mese di aprile, è avvenuta la fondazione, a Ginevra, di PKD International, organizzazione internazionale per combattere il rene policistico (Polycystic Kidney Disease, PKD) che colpisce 800.000 persone in Europa e $12 \mathrm{mi}$ lioni nel mondo. Alla Presidenza è stata nominata Tess Harris, già alla testa dell'associazione inglese per la lotta al rene policistico. AIRP è orgogliosa di essere tra le Associazioni internazio- nali che hanno contribuito alla sua fondazione, assieme a Australia, Canada, Francia, Germania e Giappone.

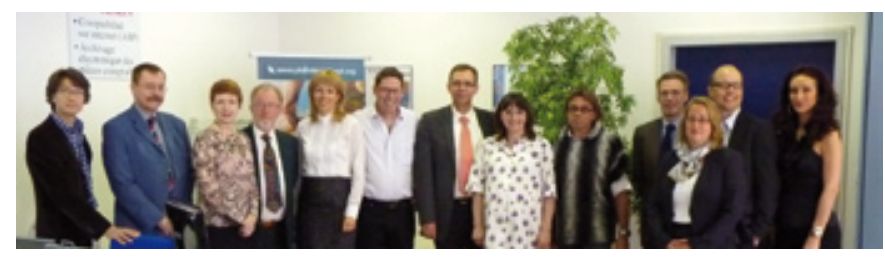

Il Board di PKD International.

Un'altra novità importante, è stato l'esordio di AIRP in TV, con la partecipazione a ben due trasmissioni. Il 22 aprile alle 18, in diretta su Telelombardia, al programma "HappyHour", durante il quale si è parlato di rene policistico e di AIRP. Una settimana dopo, il 29 aprile, alle 19, sempre in diretta su Lombardia Channel, al programma "Infopoint", anche qui per parlare di rene policistico e di AIRP. Entrambe le trasmissioni hanno visto la preziosa presenza del Professor Francesco Scolari (vi ricordo che nel sito www. renepolicistico.it/video potete trovare i link alle registrazioni video).

Il 10 e 11 maggio, invece, eravamo a Palermo, per patrocinare e partecipare a un importante Conve- 
gno presieduto dalla Dottoressa Flavia Caputo e condotto dal Dottor Vito Sparacino, che metteva intorno a un tavolo tutti i soggetti coinvolti nel lungo processo che conduce dalla dialisi al trapianto di rene, con il lungo calvario di controlli, esami, interventi, speranze, attese, delusioni e improvvisi squarci di luce. Il Convegno ha ripercorso, attraverso i diversi protagonisti coinvolti, i tre passaggi fondamentali che i pazienti attraversano: l'inserimento e il mantenimento in lista di attesa, l'intervento di trapianto, il follow-up post-trapianto.

Sempre nel mese di maggio, questa volta a Rimini, si è svolta la quinta tappa del Roadshow AIRP 2010-2012, con il $5^{\circ}$ corso di aggiornamento per Medici di Medicina Generale, Nefrologi, Biologi e Infermieri e tavola rotonda rivolta ai Pazienti. Si è parlato di genetica legata a questa malattia (Dottoressa Alessandra Boletta), di manifestazioni renali del rene policistico (Professor Francesco Scolari), di manifestazioni extrarenali (Dottor Renzo Mignani), di ipertensione arteriosa legata ad ADPKD (Dottor Alessandro Zuccalà), dei nuovi aspetti terapeutici (Dottor Riccardo Magistroni) e di rene policistico autosomico recessivo e autosomico dominante nel bambino (Dottoressa Laura Massella). E stato un dibattito intenso, concluso con una tavola rotonda, tra medici e con uno scambio tra medici e pazienti sulle problematiche personali. In particolare, è stata rivolta una calorosa esortazione ai genitori di bambini affetti da questa patologia, affinché assicurino loro la massima serenità, senza "medicalizzare" troppo la loro vita, pur rispettando l'importanza della misurazione della pressione arteriosa, che è la prima spia cui porre attenzione per la prevenzione della malattia cardiovascolare. Ai nostri ospiti, il Professor Leonardo Cagnoli e il Dottor Renzo Mignani, vanno i nostri più calorosi ringraziamenti.

I prossimi appuntamenti del Roadshow AIRP sono previsti nel prossimo mese di novembre: il 5 a Roma e il 26 a Vicenza. L'appuntamento del $1^{\circ}$ ottobre è invece ancora in via di definizione, ma vi sarà comunicato al più presto.

Anche la nostra campagna di comunicazione prosegue e miete allori. Il 31 maggio, a Milano, presso l'Auditorium del Centro Culturale San Fedele, alla cerimonia del premio Mediastars, SS\&C, l'agenzia di marketing e comunicazione di Varese, è stata premiata per la campagna pubblicitaria "Contro il Rene Policistico a Tempo di

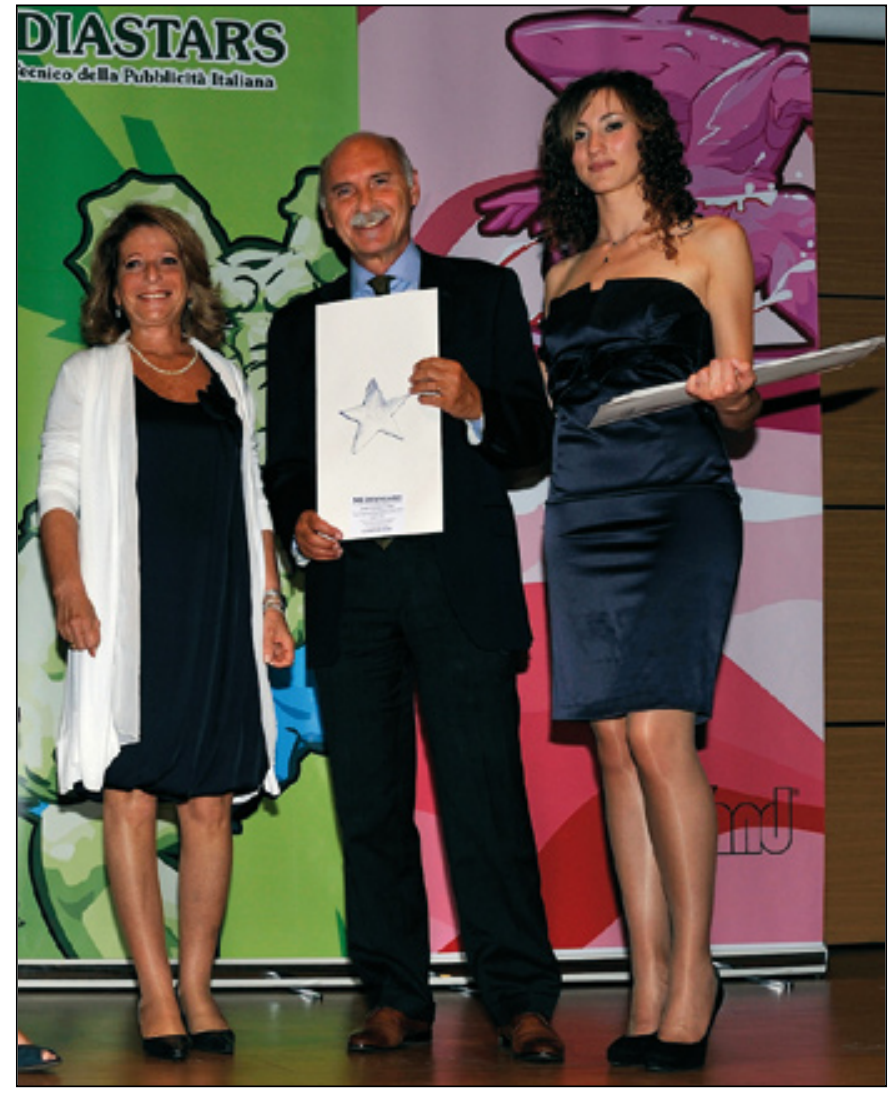

La consegna del premio Mediastars

Blues” realizzata per AIRP. L'agenzia ha ottenuto ben tre riconoscimenti per l'area audio-video: Art Direction, Copywriting e Interpretazione Audio, quest'ultimo grazie al bravissimo speaker Guido Ruberto.

Un altro riconoscimento è stato ritirato a Palermo l'11 giugno, alla cerimonia del Premio Agorà.

Prima di lasciarvi alla lettura dei contributi di questa Newsletter - l'articolo scientifico di Renzo Mignani e Leonardo Cagnoli dell'UOC di Nefrologia e Dialisi dell'Ospedale Infermi di Rimini e la rubrica "La Voce dei Pazienti” - vi ricordo che il versamento del 5x1000 alla nostra Associazione è di fondamentale importanza per rendere possibile la nostra stessa esistenza. Ecco il Codice Fiscale da indicare sulla dichiarazione dei redditi per sostenere AIRP: 97422810156.

Non perdete questa occasione di sostenere il nostro impegno e il nostro lavoro per tutti voi!

A presto 